Argumentation et Analyse du Discours

\title{
Introduction. Pour une analyse du discours de l'école. Frontières et jalons
}

\section{Pascale Delormas}

\section{OpenEdition}

1 Journals

Édition électronique

URL : http://journals.openedition.org/aad/2488

DOI : 10.4000/aad.2488

ISSN : $1565-8961$

Éditeur

Université de Tel-Aviv

Référence électronique

Pascale Delormas, « Introduction. Pour une analyse du discours de l'école. Frontières et jalons », Argumentation et Analyse du Discours [En ligne], 19 | 2017, mis en ligne le 15 octobre 2017, consulté le 23 septembre 2019. URL : http://journals.openedition.org/aad/2488; DOI : 10.4000/aad.2488

Ce document a été généré automatiquement le 23 septembre 2019.

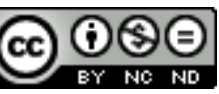

Argumentation \& analyse du discours est mis à disposition selon les termes de la licence Creative Commons Attribution - Pas d'Utilisation Commerciale - Pas de Modification 4.0 International. 


\section{Introduction. Pour une analyse du discours de l'école. Frontières et jalons}

Pascale Delormas

1 Un rappel des recherches en matière d'analyse du discours scolaire s'impose en préambule de ce dossier. En effet, dans le domaine de l'école, les travaux qui relèvent de l'analyse du discours (AD) sont rares et il faut faire état de l'intérêt que de nombreux linguistes lui ont accordé dans les années soixante-dix, mais aussi des recherches réalisées dans un passé plus récent, avant d'aborder les problématiques qui ont orienté la construction de ce numéro et qui justifient le choix des objets sélectionnés : discours institutionnels (programmes, rapports), manuels et pratiques langagières des acteurs.

\section{La recherche sur les problématiques scolaires en France : un partage disciplinaire}

2 On a coutume de considérer que les problématiques scolaires relèvent de la sociologie, des sciences de l'éducation et de la didactique. Or, comme l'affirme le plaidoyer de Sumpf et Dubois (1970 : 4) en faveur des sciences du langage, la perspective linguistique et particulièrement - nous l'ajoutons - l'approche de l'analyse du discours, s'avère ici particulièrement pertinente :

Que peut en effet apporter la linguistique à la pédagogie ? Comme la psychologie, et compte tenu du succès de ses modèles antérieurs, comme le modèle phonologique en linguistique structurale, elle peut espérer apporter une plus grande rigueur. Du fait même qu'elle n'est pas seulement une théorie de la langue, mais aussi une théorie des langues et du langage, elle peut, différemment, mais aussi légitimement que la sociologie, prétendre apporter un point de vue sinon total, du moins plus global. 

scolaire en montrant comment la sociolinguistique et la didactique se saisissent des objets qui leur sont communs.

4 Notons que la grande proximité de l'AD et de la sociolinguistique a amené les chercheurs à souligner ce qu'elles s'apportent mutuellement (Boutet et Maingueneau 2005). La « sociolinguistique scolaire» (François 1980) en fournit de bons exemples. Elle a pour objet étudiélles pratiques langagières des élèves et des enseignants (interactions verbales en classe, enquêtes sur les pratiques, sur les modalités d'évaluation, mise en œuvre des supports, ...) et pour objet visé la question des inégalités sur laquelle l'école peine à peser. Pour cela, il s'avère nécessaire de dépasser des approches considérées comme réductrices et de s'intéresser à "l'institutionnalisation de l'échec», "les pratiques pédagogiques et langagières de l'école [étant sans doute] tout à la fois cause et conséquence des caractéristiques enregistrées chez les élèves orientés " (BautierCastaing 1980: 9). En d'autres termes, il s'agit de ne pas négliger l'institution, dimension centrale dans la perspective de l'analyse du discours et de ne pas se contenter des approches sociologique et linguistique :

On peut penser que l'enseignement de la langue maternelle et ses pratiques pédagogiques restent le lieu d'intervention le plus accessible et peut-être le plus immédiatement efficace pour une réduction des échecs, ce à plusieurs conditions. Premièrement, dépasser les analyses sociologiques qui, par leur globalisation, masquent la réalité des différences de fonctionnements langagier et linguistique ; deuxièmement, dépasser l'option labovienne [...]; troisièmement, dépasser une approche strictement linguistique des différences élèves-élèves et élèves-maître pour atteindre le niveau des pratiques langagières elles-mêmes et des conceptions de la langue sous-jacentes (par analyse strictement linguistique, il faut entendre une approche de la langue essentiellement syntaxique dont on croit rendre compte à l'aide d'indices tels que la mesure de la complexité des structures, le nombre d'occurrences de certains éléments et surtout le non-dépassement de la dimension phrastique) [...].

Et lorsque l'objet étudié est l'apprentissage des usages littératiés, on a recours en outre à l'anthropologie de l'écrit (Goody 1979), considérée comme un éclairage majeur du système scolaire inégalitaire auquel sont confrontés les élèves culturellement défavorisés (Bautier 2005, Bautier et Rayou 2009, Bonnéry 2014). Bautier-Castaing (1980 : 9) note ainsi que

compte tenu des fonctions sociales et intellectuelles de l'écrit, on peut supposer que la relation du locuteur à ce système, la maîtrise qu'il en a, induit une attitude par rapport au savoir lié à la pratique de l'écrit et détermine un style cognitif spécifique, par là une appropriation des connaissances elle-même spécifique. Une première direction de recherche aurait comme but l'analyse de ces corrélations, en particulier, afin de déterminer les types d'apprentissage susceptibles de neutraliser les différences, l'étude du lien entre orientations cognitives et types de maîtrise du ou des code(s) écrit(s). (idem)

6 Parmi les objets étudiés par les sociolinguistes en rapport avec l'école, la question de la diversité linguistique est privilégiée (Blanchet 2015) : combattant un mal bien français, la glottophobie, fondée sur le fait de considérer comme inférieures certaines formes linguistiques (Blanchet 2016), Les chercheurs prônent la nécessité de prendre en considération le plurilinguisme et le discours normatif (Berrendonner 1982) comme la variation linguistique (Bachman et Basier 1984, Bertucci 2010) pour éviter qu'en soit fait un motif de discrimination.

Argumentation et Analyse du Discours, 19 | 2017 
7 La recherche en didactique ne partage pas l'objet visé par la sociolinguistique : force de proposition, voire prescriptive, elle préconise les pratiques à mettre en place. Cependant, les deux disciplines sont susceptibles de s'enrichir à travers le même objet étudié :

Prétendre enseigner le français signifie [...] tendre à la transmission de savoirs relatifs à un objet dynamique, évolutif, nécessairement hétérogène. La sociolinguistique se donne pour objectifs l'observation, la description, la compréhension, l'analyse de cette hétérogénéité. On voit dès lors que sociolinguistique et didactique ont/devraient avoir affaire ensemble. C'est ainsi que des chercheurs en didactique éclairent leurs préoccupations à la lueur de la sociolinguistique. Parallèlement, la sociolinguistique trouve dans les problématiques liées à la didactique de la langue des lieux d'application des concepts et théories qu'elle met en jeu (Guérin 2011 : 139-140).

Cependant, les propos ne sont pas toujours aussi positifs :

La sociolinguistique se flatte volontiers de sa double attache, à l'empirie des données et à des objectifs de théorisations complexes, qui relèvent à la fois des sciences du langage et de réflexions de sciences sociales et/ ou de disciplines du texte. Toutefois, il s'avère en pratique que ce n'est pas sans difficultés que cette double dépendance prend forme et tente de "faire discipline » (Gadet et Guérin $2015: 7)$.

9 Pourtant essentielle aux yeux des linguistes fondateurs de l'École française de l'analyse $\mathrm{du}$ discours, on observe que ce sont les sociolinguistes ${ }^{2}$ qui revendiquent sa vocation critique. Du fait que la sociologie de la reproduction dans le champ de l'école était largement influencée en France par les travaux de Bourdieu ${ }^{3}$, les recherches de Bernstein $^{4}$ qui liaient étroitement les inégalités scolaires au discours pédagogique n'ont pas trouvé en France l'écho qu'elles auraient mérité5. Elles n'ont été traduites en français que tardivement et ce sont des chercheurs en sciences de l'éducation (en particulier les membres du groupe Escol) qui ont contribué à le faire connaître (Bautier 2001). Cependant les difficultés d'ordre épistémologique que l'on examinera plus bas comptent pour beaucoup dans l'abandon de ce terrain.

Quant au discours scolaire, on remarque que rares sont les chercheurs en sciences du langage qui s'y intéressent aujourd'hui en France. Alors que l'AD rencontre un succès certain lorsqu'il s'agit des discours ordinaires, les discours de l'école n'ont pas été examinés explicitement selon cette perspective depuis longtemps - ceci pour des raisons qu'il serait d'ailleurs intéressant d'examiner, de façon réflexive, comme un indice de positionnement dans le champ de la recherche. En outre, du fait que les sciences du langage sont perçues comme une discipline contributive à la recherche en didactique et en sciences de l'éducation, une certaine confusion s'est instaurée. Pourtant l'analyse du discours scolaire ne se réduit pas à l'approche énonciative, ni à l'analyse des interactions verbales (entre enseignants et apprenants et entre apprenants), ni à celle des pratiques ${ }^{6}$. Ce constat devrait encourager les chercheurs à en clarifier la spécificité?

\section{Des questionnements d'ordre épistémologique}

Les analystes du discours scolaire se heurtent à plusieurs obstacles épistémologiques. Nous en retiendrons trois: l'hétérogénéité constitutive des corpus, la dimension institutionnelle, la perspective idéologique. 
Les contributeurs du numéro 5 de Langue française consacré aux relations entre linguistique et pédagogie soulignent la difficulté de circonscrire le domaine d'étude de l'analyse du discours, étant donné le caractère inextricable de la relation qu'entretiennent discours éducatif et discours philosophique. Ainsi Sumpf (1970: 23-24) écrit-il :

En fait, qu'est-ce qui n'est pas l'homme? Nous nous trouvons [...] devant un domaine dont les objets sont par principe, indéfiniment substituables les uns aux autres puisque rien n'est absolument l'homme. Nous nous trouvons devant un domaine opaque (contenu dans l'homme) et du coup tout performatif interfère avec un discours autre (religieux, philosophique, etc.) qui est toujours un discours sur l'homme. C'est dire que toute question sur l'éducation porte en elle un écart indéfini entre les normes du performatif (je vous engage à faire $\mathrm{X}$ ) et les normes du domaine ( $\mathrm{X}$ est tout l'homme). L'analyse des discours sur l'éducation part nécessairement des différentes formes de performatif pour aller vers le discours sur l'homme (compte tenu de l'axe philosophique-rhétorique).

Les corpus sont nécessairement hétérogènes et mouvants et l'on conçoit que «l'on puisse s'effrayer du disparate de la collection des textes; [car] celle-ci se caractérise non seulement par son extrême diversité, mais encore par son instabilité fondamentale » (Sumpf et Dubois, $70: 4$ ). La diversité et la mobilité ne sont cependant pas considérées comme des obstacles dès lors que l'on admet que la circulation des discours et l'interdiscursivité sont des paramètres incontournables pour qui pratique l'AD, comme en témoignent Désirat et Hordé (1977: 3-4), dans l'introduction du numéro 45 de la revue Langages (1977) consacré à la «Formation des discours pédagogiques » :

Les collaborateurs de ce numéro de Langages n'ont pas voulu éviter la nécessaire réflexion sur les modes d'approche du discours. Chacun, à sa façon, a cherché, contre la reconduction de la notion d'écart, à établir non la spécificité d'un «discours pédagogique», mais plutôt les formes d'échanges entre discours hétérogènes, entre discours et pratiques sociales.

Dès lors que l'on cherche à prendre en compte l'épaisseur discursive des énoncés, s'impose la nécessité d'analyser les discours de l'institution dont ils adviennent, qui les rendent possibles et qui les portent. La plupart des articles du numéro de Langages propose des analyses linguistiques des discours de l'école envisagée comme institution. Le questionnement annoncé par les deux responsables de cette publication pourrait être formulé de la même façon ici :

Quelles relations peut-on décrire entre discours fondateurs et discours de fonctionnement de l'institution (discours institutionalisant/institutionalisé) si l'on admet que l'un et l'autre sont en interaction selon un axe orienté de l'un à l'autre : décision $=>$ application, sauf à laisser échapper le déplacement des effets de sens produits par l'action engagée ? C'est, à notre avis, ces échanges qui sont occultés par des distinctions comme discours politiques/discours pédagogiques. (Désirat et Hordé, idem)

Or la crainte de ne pouvoir appréhender les discours institutionnels du fait de leur extrême mobilité amène certains à y renoncer au bénéfice d'objets apparemment plus stables, comme celui de la place octroyée à la linguistique dans les manuels et dans les pratiques. On peut lire les arguments suivants dans la présentation du numéro 55 de Langue française :

Le risque de décrire une situation en voie d'être périmée ou de paraitre nous substituer à tous les organes (corporatifs, syndicaux, gouvernementaux) qui, présentement, réfléchissent aux formes et contenus des formations nous a conduits 
à ne pas privilégier le terrain institutionnel comme source d'enquête pour une " sociologie » des pratiques d'enseignement, ce qui, en outre, n'est ni notre objet ni la vocation de Langue française.

C'est donc sur la base d'une recherche des problèmes spécifiques au rôle de la linguistique dans la didactique du français que J. Hébrard, F. Gadet et F. Maziere, D. Campagne et $\mathrm{M}$. Verdelhan ont examiné les ouvrages destinés à la formation des enseignants, les revues à destination pédagogique, les concepts à l'œuvre aujourd'hui dans la formation des enseignants de français langue étrangère, les expériences de recyclage en linguistique d'enseignants du primaire et du secondaire. (Chiss $1982: 3-4$ )

La perspective de l'analyse du discours suppose de tenir les deux bouts de la chaîne, c'est- à-dire de ne pas déconnecter idéologie et spécificité énonciative du propos. Il s'agit de faire intervenir «l'ordre idéologique " pour reprendre la formule de Hailon (2014 : 33). C'est ce que nous tentons de faire, dans la droite ligne de Bréal (1872), à propos duquel Delesalle (1977 : 83) fait le commentaire suivant :

Plus généralement, ce texte [de Bréal] nous fait nous interroger sur ce qu'est un discours pédagogique : les textes de cette nature, qui traitent de l'enseignable, sont toujours tirés entre le scientifique et le politique, la réduction du premier s'opérant dans la reconnaissance ou l'effacement du second. Celui-ci tire une partie de sa force d'une poussée irrésistible du scientifique et d'un affleurement inégal du politique refoulé. Les deux sont bien là, s'ils ne sont pas au même niveau, et il faut toujours les lire à travers ce qui se donne comme pédagogique, et surtout à travers ce qui se donne - c'est le cas ici - comme pédagogique par excellence, c'est-à-dire le discours même de la violence symbolique qui pose le bienfait du savoir comme un axiome et rejette dans l'ombre ses buts et son rôle.

\section{Des notions clés : « didacticité » et « interdiscours »}

Si l'investigation d'une notion comme celle de «didacticité » relève moins du souci de faire apparaitre la teneur idéologique des discours que de celui de décrire leurs caractéristiques linguistiques intrinsèques, elle réfère en dernière instance à la capacité des individus de transmettre des valeurs dans un cadre culturel, selon des normes et un projet sociétal qui les dépassent.

Les recherches de Jean Peytard (Peytard 1983, 1992, Peytard et Moirand 1992, Cortès et Peytard 1988) ${ }^{8}$, qui associèrent analyse du discours, sémiotique et didactique - et dans un second temps l'informatique -, marquèrent une avancée majeure dans le domaine de l'analyse du discours de l'école. Les commentaires soulignent «son intérêt pour la dimension sociale et idéologique, [qui] en fait un promoteur de l'analyse des discours » (Madini 2010). On peut lire dans les actes du colloque Miroir qui lui fut consacré en 2012 qu'il

n'aura pas peu contribué à promouvoir, en la structurant, la "pensée didacticienne » au sein des sciences du langage, de par les discours de surface qui attestent de sa légitimité scientifique et de sa vitalité fonctionnelle en tant que sphère d'observation, d'expérimentation et d'intervention. Il était donc plus que temps de rendre hommage à celui que d'aucuns s'accordent désormais à considérer comme un des précurseurs de l'analyse de discours et de la didactique des langues (Borg 2012 : 273).

Dans les années 90, les premiers numéros de la revue Les Carnets du Cediscor ${ }^{9}$, poursuivant les travaux de J. Peytard, témoignent de l'intérêt accordé à la notion de didacticité mais sans la référer au discours de l'école. Ainsi le texte inaugural du 
premier numéro est-il consacré à «Un lieu d'inscription de la didacticité. Les catastrophes naturelles dans la presse quotidienne" - car comme l'affirment explicitement Beacco et Moirand (1993), «l'un des axes de recherche prioritaire du Cediscor a été, durant l'année 1990, de s'interroger sur la notion de didacticité à travers l'étude de ses lieux d'inscription dans les discours ordinaires et les discours médiatiques ». Lorsque Moirand (1993) et Beacco et Moirand (1995b) se consacrent à l'analyse des "discours de transmission des connaissances ", c'est toujours avec l'idée de promouvoir la notion heuristique de "didacticité ",

forgée pour les besoins de la recherche, elle a permis de se démarquer des travaux antérieurs (sur les langues de spécialité d'une part, sur les discours politiques de l'autre) et, par suite, de collationner des textes, dont les déterminations sont floues, et constitutivement hétérogènes, d'entrer dans des discours, que l'on peut qualifier d'ordinaires, parce qu'ils n'ont pas pour vocation première de transmettre des connaissances (discours médiatiques, brochures ou guides touristiques, notices de catalogues d'exposition...), d'aborder le discours (objet théorique) et les documents (objets empiriques) au travers de notions permettant de décrire les dimensions cognitives et interactionnelles de la didacticité, telles qu'elles s'inscrivent dans la matérialité des textes (Beacco et Moirand 1995b:33).

Le numéro 2 des Carnets $d u$ Cediscor "Discours d'enseignement et discours médiatiques » a pour projet d'étayer cette notion de didacticité, comme l'indique le sous-titre « Pour une recherche de la didacticité » à travers l'analyse de deux types de discours, les discours d'enseignement relevant d'une catégorie générique plus englobante que celle de "discours pédagogique ». La présentation du premier dossier (Cicurel 1994) met en avant la nécessité de prendre en compte, outre le dialogisme interne, la perméabilité des textes à visée didactique :

[ils] ne sont pas à l'intérieur d'un univers clos, replié sur lui-même, mais bien au contraire ils sont comparables à des terrains éminemment perméables. Ils ne peuvent être dissociés d'un « extérieur ». Extériorité formée d'une constellation de variables accompagnant la production du discours d'enseignement: relation enseignant/apprenant; discipline enseignée; représentations de l'idéal du moiélève ou du moi-professeur; proximité avec la conversation ordinaire qui interrompt le dialogue didactique ; jeu avec l'irruption d'événements extérieurs au cours (Cicurel op. cit. : 15).

21 L'avant-propos du numéro 3 des Carnets du Cediscor (Beacco et Moirand 1995a), consacré aux « enjeux des discours spécialisés ", va dans le même sens et s'inscrit explicitement dans l'approche française de l'analyse de discours. Il s'agit de s'interroger sur la didacticité des discours qui transmettent de l'information, à partir d'une description systématique des traces qui l'actualisent dans la matérialité des textes sans négliger l'importance d'un hors-texte, l'interdiscours se manifestant à tous les niveaux d'expression verbale. L'objectif est de

décrire linguistiquement des discours "spécialisés » ou lieux de transmission de connaissances et [ $d$ ']adosser les régularités langagières mises en évidence à un endehors de la discursivité (cognition, institution, représentations sociales...) susceptible d'en rendre compte et d'alimenter en retour ces descriptions des fonctionnements discursifs. En somme, et malgré les apparences, la problématique fondatrice de « l'École française d'analyse du discours » (Beacco et Moirand op. cit.: 1).

Enfin, dès le numéro 4 des Cahiers du Cediscor (1996), les auteurs prennent une nouvelle orientation qu'on ne peut confondre avec une approche discursive puisqu'ils 
s'attachent à décrire les «spécificités énonciatives » dans "l'interaction en classe de langue » et les « stratégies d'enseignement » en lien avec l'acquisition ${ }^{10}$.

Aujourd'hui, quelques équipes de recherche manifestent leur engagement en faveur de l'AD. L'énoncé programmatique de l'équipe EDA (Éducation, discours, apprentissage Université Paris 5) annonce que ses travaux « convoquent de l'analyse de discours $»^{11}$. L'équipe LAB-E3D (Université de Bordeaux), sans la nommer explicitement, fait appel aux notions de «contexte", de "positionnement», de «communauté discursive " (Jaubert, Rebière et Bernié 2004) ${ }^{12}$, autant de termes issus de l'AD.

\section{Les genres de discours abordés}

Les articles présentés dans ce numéro explorent à nouveaux frais des discours déjà abordés par le passé, qu'il s'agisse de textes de cadrage en matière de politique éducative (programmes et rapports), de manuels, d'exercices scolaires ou des dires et écrits professionnels des enseignants. L'état des lieux est forcément lacunaire mais on verra que les outils mis en œuvre et les analyses correspondent à une conception partagée du discours scolaire comme formation discursive. Le poids accordé à la notion de genre dans l'approche discursive nous conduit à regrouper les contributions en fonction des caractéristiques génériques des discours étudiés. On peut les regrouper selon trois catégories, celles du "champ discursif », de la "sphère d'activité », et du «lieu d'activité » (Maingueneau 2016: 9). Les discours institutionnels relèvent de la première catégorie (genres associés à un positionnement), dans la mesure où ils sont mobilisés dans le cadre de controverses et/ou d'orientations éventuellement en concurrence - par exemple lorsqu'il s'agit d'introduire des pratiques innovantes. L'intergénéricité caractéristique des manuels et les spécificités culturelles sur lesquels ils reposent conduisent à les rattacher à la seconde catégorie - dans une perspective foucaldienne très englobante, sans restriction aux lieux désignés comme scolaires, étant donné l'interaction permanente avec des discours extérieurs au domaine scolaire. Les exercices imposés aux élèves, les propos déclaratifs des enseignants et leurs routines relèvent de la troisième catégorie : ils sont pratiqués dans l'espace de la classe.

\subsection{Analyse des discours institutionnels}

Le numéro 101-102 de Pratiques, «Textes officiels et enseignement du français » (1999), rassemble des articles sur les contenus des programmes pour tenter «d'objectiver la connaissance de la discipline telle que les discours officiels la configurent» (Petitjean : 7). Pour mieux comprendre les « enjeux discursifs des Instructions officielles ", Paveau analyse l'ethos discursif de ces textes pour faire émerger l'image des acteurs institutionnels et des destinataires. Borowski, dans le même numéro, analyse les enjeux pragmatiques, c'est-à-dire les visées de l'énonciateur et les stratégies discursives, dans la brochure La maitrise de la langue à l'école. Reboul-Touré, quant à elle, montre à travers le changement de statut du concept de discours, que les enjeux informatifs et les visées didactiques complexifient les rapports de didacticité dans les Instructions officielles des collèges.

Cependant Petitjean (1999: 5) déplore que le point de vue adopté soit un point de vue interne, 
au détriment d'un rapprochement avec d'autres textes (débats parlementaires, campagnes de presse, revues pédagogiques, discours théoriques, manuels, travaux d'élèves...) qui permettraient de contextualiser les textes officiels et de mieux comprendre leur logique et leur axiologie » et que manquent les témoignages d'acteurs (rédacteurs des I.O. et enseignants).

L'analyse du dispositif énonciatif peut être directement articulé à la subjectivité des acteurs. Ainsi, Désirat (1977 : 17) est amené à souligner le hiatus entre injonction et incorporation par les praticiens des directives :

[Les programmes] sont le nœud central de l'acte/discours pédagogique, le centre de son ambiguité, et par cela même l'objet d'effacements systématiques. Ce qui est le plus régulièrement occulté dans l'acte pédagogique proprement dit, c'est justement l'origine de la parole, alors qu'ici elle exhibe ses masques. On voit mieux aujourd'hui les raisons de cette omission: l'enseignant croit-il ce qu'il profère? Peut-il s'identifier à la source du savoir qu'il diffuse, alors qu'il est le plus souvent loin des lieux où les sciences s'élaborent, loin du temps où elles ont proposé ce qu'il enseigne aujourd'hui? N'est-il pas hanté par l'espace qui sépare le contenu "scientifique» des disciplines et le discours "élémentaire » qu'il est chargé de tenir?

D'un autre côté, ou du même, s'identifie-t-il au pouvoir politique qui l'investit de l'autorité nécessaire à l'acte pédagogique, au pouvoir qui l'A choisi, sélectionné, formé, et détermine les objectifs de son travail ? révèlent et configurent la conception du métier, ainsi Dubois et Sumpf (1970:27-28) affirment que :

[1]es concours de recrutement des professeurs du second degré font partie de l'institution scolaire puisqu'ils sélectionnent les candidats licenciés qui, par une série d'épreuves, manifestent la maîtrise d'un modèle implicite de l'enseignement français du français. Les rapports des jurys sont eux-mêmes une institution, leur obligation relevant d'une nécessité à la fois théorique et morale. Il s'agit en effet de fournir des données chiffrées qui, d'une certaine manière, prétendent offrir un état de l'intelligence française et, par ailleurs, ce jugement doit comporter des attendus, c'est-à-dire les justifications des sanctions prononcées.

Les rapports sont donc des textes et ressortissent à l'analyse de contenu et à l'analyse de discours. On doit pouvoir répondre aux questions que l'on est, comme candidat, en droit de se poser : que veut le jury? que faut-il faire et ne pas faire? quel est le champ des possibles et celui des interdits? Et c'est dans la mesure où la réponse répond à un modèle culturel qui est aussi celui des candidats que ces derniers lisent les rapports d'agrégation et s'y retrouvent.

Dans ce numéro, l'article de Pascale Delormas, «De la contestation doxique dans le champ discursif de l'école française ", poursuit un vaste projet d'analyse des discours institutionnels (Delormas 2010) à travers des exemples de controverses qui traversent le champ de l'institution scolaire à différents niveaux de diffusion de la doxa. Les contributions de Xavier Levoin et de Marlène Loicq s'inscrivent dans cette ligne lorsqu'ils s'emparent de la question spécifique de l'interdiscours en matière de politique d'éducation au numérique et aux médias. Dans son article "Numérique éducatif et interdiscursivité : comment la circulation de mots d'ordre contribue à la mobilisation des acteurs", Levoin examine le discours d'autorité que constitue une argumentation fondée sur l'articulation du discours de l'industrie au discours institutionnel et dont la visée est la promotion des outils numériques dans les pratiques enseignantes. Loicq, dans "L'analyse de discours institutionnels sur l'éducation aux médias. Approche socio-anthropologique et comparative », propose d'aborder par la 
comparaison l'influence exercée par les médias dans l'élaboration des Programmes d'éducation aux médias en Australie, au Québec et en France.

\subsection{Analyse des manuels}

30 Le second volet de ce numéro est consacré à la relation qu'il s'agit d'établir entre manuels et institution. À la question de savoir ce qui fait qu'un texte devient un instrument de formation, la recherche d'indices linguistiques de didacticité n'est pas la seule réponse. L'examen de la spécificité fonctionnelle de ce genre de discours permet d'en comprendre la complexité. Il est à la fois outil, thésaurus et vecteur d'idéologie.

31 Tributaire d'un discours autre du fait de son hétérogénéité énonciative et générique et du décalage entre les codes du savoir savant, de la discipline et de son enseignement auxquels il se réfère (Hébrard 1982, Delormas 2017), le manuel suppose la médiation d'un tiers; il requiert une mise en œuvre pédagogique et c'est l'existence de l'usage potentiel qu'en font les praticiens, dans la classe, en interaction avec les élèves, qui le justifie.

32 Le manuel apparaît comme "le point de focalisation des valeurs essentielles que transmet l'institution scolaire» (Choppin $1980: 2$ ). Les analystes du discours mettent en avant le fait que ces dispositifs façonnent savoirs et apprentissages selon une circularité qui ramène à l'institution et qui permet de parler de "discours de scolarisation » (Verdelhan-Bourgade $2002: 1-2)^{13}$, le discours du manuel renvoyant sans cesse à l'École comme évidence (Maingueneau 1979, 1983) ${ }^{14}$. Ce jugement constitue un préambule à l'analyse discursive.

Considéré comme un métadiscours qui obéit aux orientations des programmes et dont la visée est de fournir aux enseignants et aux élèves le moyen de construire un discours partagé, le manuel «ne représente pas le discours que l'on tient dans la classe de français [par exemple], mais réunit un ensemble d'énoncés destinés à faire tenir le discours sur la littérature " (Fressange 1970: 45). Dans son article "Circulation et modélisation du discours testimonial dans le champ scolaire : l'exemple des manuels » publié dans ce numéro, Olivia Lewi envisage le manuel dans sa fonction modélisatrice. Le cas de témoignages de la Shoah permet de montrer comment s'articulent les discours disciplinaires d'un manuel à l'autre : le manuel littéraire fournit des canevas textuels et énonciatifs aux scripteurs ordinaires et, selon un mouvement symétrique, leurs témoignages s'imposent comme exemples canoniques dans les manuels d'histoire. La contribution de Patricia von Münchow, "Quel rapport entre passé et présent dans les manuels scolaires d'histoire en France et en Allemagne?", montre à travers une comparaison interculturelle que les choix des manuels édités en deçà et au-delà du Rhin sont révélateurs des points de vue nationaux très différents quant à la transmission de la connaissance des événements de la guerre de 14. Les manuels allemands font appel à la connaissance sensible des élèves qu'il s'agit d'informer et d'éduquer dans une perspective contemporaine, en rapport avec la conscience politique du futur citoyen; les manuels français ne présentent pas l'événement de la même façon en raison de l'issue de la guerre et de la perception contemporaine qu'en a la population. 


\subsection{Analyse des pratiques discursives des élèves et des enseignants}

Le troisième volet de ce dossier interroge les pratiques discursives des élèves et des enseignants. Les exercices demandés aux élèves du fait de l'influence qu'ils exercent sur la formation de l'individu et du citoyen revêtent une importance majeure. C'est ce que fait apparaître l'article de Luc Thominette, "La scénographie du yilùnwén au prisme de la dissertation. Argumentation scolaire et socialisation politique en Chine et en France ». Cette analyse rend compte de la grande diversité des modalités argumentatives qui s'imposent dans les curricula en fonction des traditions scripturales comme en atteste la comparaison de deux genres discursifs en vigueur dans deux univers culturels contrastés.

Didier Colin, dans son article « Recherche et institution en concurrence. L'exemple de la conception de l'enseignement de l'écriture dans les dires enseignants ", examine le degré d'assimilation des textes prescripteurs dans les pratiques effectives à travers l'identification de l'appropriation-reformulation des prescriptions institutionnelles et de leur transposition. Il rejoint en cela le projet de Normand (1982: 26) à propos de l'enseignement de la grammaire ainsi formulé : "à partir de quelques ouvrages dits de grammaire, [il s'agit d'] essayer de montrer "ce qui passe" des recherches linguistiques contemporaines, dans l'application à un problème grammatical traditionnel, et comment ».

7 Aurore Promonet, dans son article «Les traces écrites scolaires : une cristallisation de discours ", aborde, elle aussi, la question de la circulation des discours institutionnels dans l'espace de la classe à travers une démarche d'enquête et d'observation auprès des professionnels de l'enseignement. Elle propose une typologie des actes discursifs des acteurs repérables dans la chaîne des écrits qui jalonnent les séances d'enseignement pour appréhender le niveau de monstration de l'action conjointe enseignant/élève en fonction du moment du cursus scolaire. En cela, elle prend en considération la place et les pratiques discursives des élèves.

Consciente de l'insuffisance du panorama offert $\mathrm{ici}^{15}$, nous espérons cependant que cette exploration des frontières de l'AD scolaire fournira aux lecteurs des indications qui pourront leur permettre d'évoluer plus facilement dans le dédale disciplinaire observable en France en la matière. Les analyses présentées ici renvoient à des notions fondamentales pour l'AD; elles constituent autant d'exemples qui rendent compte de l'intérêt de poursuivre l'investigation à travers leur mise en œuvre.

\section{BIBLIOGRAPHIE}

Bachmann, Christian \& Luc Basier. 1984. «Le verlan : argot d'école ou langue des Keums? », Mots 8, «L'Autre, l'Étranger, présence et exclusion dans le discours », 169-187 
Bautier-Castaing, Élisabeth. 1980. «Pratiques linguistiques, discursives, pédagogiques : cause ou conséquence de l'échec scolaire? » Langages 59, « Conduites langagières et sociolinguistiques scolaire ", 9-24

Bautier, Élisabeth. 2001. « Note de synthèse. Pratiques langagières et scolarisation » Revue Française de Pédagogie 137, 117-161

Bautier, Elisabeth. 2005. « Mobilisation de soi, exigences langagières scolaires et processus de différenciation », Langage et société 111, 51-71

Bautier, Elisabeth \& Patrick Rayou. 2009. Les inégalités d'apprentissage. Programmes, pratiques et malentendus scolaires (Paris : PUF)

Beacco, Jean-Claude \& Sophie Moirand. 1993. « Présentation », Les Carnets du Cediscor 1, 7

Beacco, Jean-Claude \& Sophie Moirand. 1995a. « Avant-propos », Les Carnets du Cediscor 3, « Les enjeux des discours spécialisés ", 9-10

Beacco, Jean-Claude \& Sophie Moirand. 1995b. « Autour des discours de transmission des connaissances, Langages 117, « Les analyses du discours en France », 32-53

Berrendonner, Alain. 1982. L'Éternel grammairien : étude du discours normatif (Berlin : P. Lang)

Bertucci, Marie-Madeleine. 2010. «Des langues des élèves à la langue de la scolarisation : quelques propositions pour l'enseignement/apprentissage du français dans un contexte plurilingue et variationniste ». Bertucci, Marie-Madeleine \& Isabelle Boyer (dir.), Transfert des savoirs et apprentissage en situation interculturelle et plurilingue (Paris : L'Harmattan), 73-98 Bonnéry, Stéphane. 2007. Comprendre l'échec scolaire. Élèves en difficultés et dispositifs pédagogiques (Paris : La Dispute)

Blanchet, Philippe (éd.). 2015. «Présentation : ce que l'étude de la diversité sociolinguistique apporte à l'étude de l'éducation aux langues, en langues et par les langues », Cahiers de Linguistique 41, « Sociolinguistique et éducation. Contribution au repérage du champ avec exemples de diversités linguistiques sur des terrains variés »

Blanchet, Philippe. 2016. Discriminations : combattre la glottophobie (Paris : Textuel)

Borg, Serge. 2012. «Analyses de discours et “pensée didacticienne”. Les lieux féconds d'une rencontre ». Synergies Monde, Gerflint, Un hommage à Jean Peytard, précurseur de la linguistique du discours et de la didactique des langues, 263-275

Borowski, Patrick. 1999. « Portraits discursifs du lectorat de la brochure "La maîtrise de la langue à l'école”, Pratiques 101-102, « Textes officiels et enseignement du français », 21-40

Boutet, Josiane. 2017. « La pensée critique dans la sociolinguistique en France », Langage et société 160-161, 23-42

Boutet, Josiane \& Dominique Maingueneau. 2005. «Présentation », Langage et Société 114, 9-13

Bréal, Michel. 1872. Quelques mots sur l'instruction publique en France (Paris : Hachette)

Bucheton, Dominique \& Yves Soulé. 2009. « Les gestes professionnels et le jeu des postures de l'enseignant dans la classe : un multi-agenda de préoccupations enchâssées », Éducation et didactique 3, 29-48

Chiss, Jean-Louis. 1982. «Présentation », Langue française 55, « Linguistique et formation des enseignants de français », 3-4

Choppin, Alain. 1992. Les manuels scolaires : histoire et actualité (Paris : Hachette) 
Cicurel, Francine. 1994. « Variables discursives dans l'enseignement des langues », Les Carnets du Cediscor 2, 13-15

Cortès, Jean \& Jean Peytard (éds). 1988. Cahiers du français des années 80, 3 « Sociolinguistique : évaluation et analyses de discours » (Paris : Didier).

Delesalle, Simone. 1977. « Michel Bréal : philologie, instruction et pouvoir », Langages 45, 67-83

Delormas, Pascale. 2010. « Analyse du discours institutionnel de l'école. Le cas de l'écriture de soi », Cahiers de sociolinguistique, 109-121

Delormas, Pascale. 2017. « L'anthologie littéraire : un genre didactique citationnel », Le discours rapporté. Temporalité, histoire, mémoire et patrimoine discursif (Paris : Classiques Garnier)

Désirat, Claude. 1977. «Les récits d'une fondation : la loi et la pédagogie ». Langages 45, 9-41

Désirat, Claude \& Tristan Hordé. 1977. Langages 45, « Formation des discours pédagogiques », 3-8

Dubois, Jean \& Joseph Sumpf. 1970. « Un modèle d'enseignement du français : analyse linguistique des rapports d'agrégation et du CAPES », Langue française 5, 27-44

Fegter, Susanne, Fabian Kessl, Antje Langer, Marion Ott, Daniela Rothe \& Daniel Wrana (éds).

2015. Erziehungswissenschaftliche Diskursforschung. Empirische Analysen zu Bildungs- und Erziehungsverhältnissen (Wiesbaden : VS Verlag)

François, Frédéric (dir.). 1980. « Conduites langagières et sociolinguistique scolaire », Langages 59 Fressange, Guy. 1970. « Le discours didactique dans les manuels de morceaux choisis de français. ", Langue française 5, « Linguistique et pédagogie », 45-69

Gadet, Françoise \& Emmanuelle Guérin. 2015. « Introduction », Langage et société 154, 7-15

Goody, Jack. 1979. La raison graphique (Paris : Minuit)

Guérin, Emmanuelle. 2011. «Sociolinguistique et didactique du français : une interaction nécessaire ", Le français aujourd'hui 139-144

Hailon, Fred. 2014. L'ordre idéologique. Éléments de cognition politique (Paris : L'Harmattan)

Hambye Philippe. 2017. « Langues et discours comme objets sociologiques : une illustration en sociologie de l'éducation », Langage et société 160-161, 59-74

Hébrard, Jean. 1982. « De curieux textes : les ouvrages destinés à la formation des enseignants. Méthodes d'analyse et analyses de leurs conditions d'énonciation et de réception ». Langue française $55,66-79$

Jaubert, Martine, Maryse Rebière \& Jean-Paul Bernié. 2004. « Significations et développement : quelles « communautés »? ", Moro, Christiane \& René Rickenmann (éds) Situation éducative et significations (Louvain-la-Neuve : De Boeck), 85-104

Madini, Mongi. 2010. «Quelques "lieux de rencontre" de Jean Peytard », Semen 29, 17-39

Maingueneau, Dominique. 1979. Les livres d'école de la République, 1870-1914, Discours et idéologie (Paris : Sycomore)

Maingueneau, Dominique. 1983. «Discours scolaire et efficacité idéologique », Dortmunder Arbeiten zur Schulgeschichte und zur historischen Didaktik, 95-100

Maingueneau, Dominique. 2003. « Les apports de l'analyse du discours à la didactique de la littérature ", Le français aujourd'hui 141,73-82 
Maingueneau, Dominique. 2016. «Quelques remarques sur la notion de genre scolaire». Recherches 65, « Genres scolaires », 7-16

Moirand, Sophie \& Jean Peytard. 1992. Discours et enseignement du français (Paris : Hachette) Moirand, Sophie. 1993. « Autour de la notion de didacticité », Les Carnets du Cediscor 1, 9-20 Moirand, Sophie. 2012. «Entre altération et reformulation, quelle place faire au dialogisme de Bakhtine dans les travaux de Jean Peytard? ». Synergies Monde, Gerflint, « Un hommage à Jean Peytard, précurseur de la linguistique du discours et de la didactique des langues », 205-230

(von) Münchow, Patricia. 2015. « Analyse du discours et éducation : territoires, extérieurs et réseaux ». Canut, Cécile \& Patricia von Münchow (éds). Le langage en sciences humaines et sociales, (Limoges : Lambert Lucas), 155-168

(von) Münchow, Patricia. 2014. «L'analyse du discours contrastive : comparer des cultures discursives ». Grezka, Aude, Malory Leclère \& Malika Temmar (éds). Les Sciences du langage en Europe : tendances actuelles. Actes du colloque 2011 de l'ASL-Association des Sciences du Langage (Limoges : Lambert-Lucas), 75-92

Neveu, Franck \& Peter Lauwers. 2007. « La notion de "tradition grammaticale" et son usage en linguistique française ", Langages 3, 7-26

Normand, Claudine. 1982. « Comment l'article cessa d'être un « petit mot ». Langue française 55, « Linguistique et formation des enseignants de français », 25-44

Paveau, Marie-Anne. 1999. « Le discours des Instructions officielles au lycée en 1995 : jeux et enjeux énonciatifs », Pratiques 101-102, « Textes officiels et enseignement du français », 10-20 Petitjean, André. 1999. Pratiques 101-102, « Textes officiels et enseignement du français », 3-7 Peytard, Jean (éd). 1983. Semen, 1. « Lecture et lecteur»

Peytard. Jean. 1992. Syntagmes 4, de l'évaluation et de l'altération des discours (sémiotique, didactique, informatique) (Besançon : PUFC)

Peytard, Jean \& Sophie Moirand. 1992. Discours et enseignement du français. Les lieux d'une rencontre (Paris : Hachette)

Reboul-Touré Sandrine. 1999. « Eléments de didacticité dans les Instructions officielles de collège : autour de la notion de discours », Pratiques 101-102, « Textes officiels et enseignement du français », 47-58

Sumpf, Joseph. 1970. «Introduction à une recherche linguistique en pédagogie », Langue française 5 , « Linguistique et pédagogie », 17-26

Sumpf, Joseph \& Jean Dubois. 1970. « Linguistique et pédagogie ». Langue française 5, 3-16

Verdelhan-Bourgade, Michèle. 2002. « Le manuel comme discours de scolarisation ", Ela. Études de linguistique appliquée 125, 37-52

\section{NOTES}

1. Cf. La distinction heuristique "objet étudié et objet spécifique ou visé » est reprise à Hjemslev par Boutet (2017): «L'objet étudié, c'est celui que le chercheur se construit au moyen de méthodes de recueil spécifiques : ce peuvent être des phrases agencées en corpus, des énoncés, des interactions verbales, des dialogues professionnels, des textes écrits, etc. L'objet spécifique, c'est celui que vise le chercheur au travers ou au moyen de cet objet ». 
2. En Allemagne, ce sont les sociologues de l'éducation qui se sont emparé explicitement des notions de l'AD. Voir par exemple Fegter, Susanne et al. (éds) ( 2015).

3. Cf. par exemple Bourdieu, Pierre. 2001. Langage et pouvoir symbolique (Paris : Seuil).

4. Il s'agissait pour Bernstein de relier la dimension langagière des processus éducatifs de l'école à la question des relations de pouvoir et de classes. Voir Bernstein, Basil. 1990. Class, codes and control, vol. 4 (Londres : Routledge) ; 1996. Pedagogy, symbolic control and identity: theory, research, critique (Londres: Taylor et Francis). Pour une synthèse, voir Sadovnik Alan R. 2001. «Basil Bernstein (1924-2000) ». Perspectives : revue trimestrielle d'éducation comparée 4, 715-731.

5. Ils connaissent leurs travaux respectifs et ils les soumettent à critique : Bernstein affirme que les théories comme celles de Bourdieu «visent à comprendre comment les relations de pouvoir extérieures sont transmises par le système... [non pas] avec la désignation du vecteur, mais uniquement avec un diagnostic de sa pathologie» (Bernstein 1990: 172). Pour Bourdieu, Bernstein aurait décrit les propriétés du code élaboré sans relier ce produit social aux conditions sociales de sa production et de sa reproduction.

6. Les linguistes et didacticiens de l'équipe Lidilem (Université de Grenoble, http://lidilem.ugrenoble3.fr) sont proches de l'AD mais l'objet étudié est l'écrit dans l'enseignement supérieur (Boch, Grossmann, Rinck). Les membres du Lirdef (Université de Montpellier) et en particulier l'équipe Alpha (http://lirdef.edu.umontpellier.fr/equipes/alpha/) travaillent selon une approche énonciative sur l'agir professionnel en didactique - Bucheton, pourtant très proche de l'AD, ne s'y réfère pas (https://educationdidactique.revues.org/543?lang=es\#tocto1n1), pas plus que Daunay, Delcambre, Denizot, Laborde-Milaa, Lahanier-Reuter, Reuter, membres de l'équipe Théodile (Université de Lille, http://theodile.recherche.univ-lille3.fr/cms/index.php? option=com_content\&id=564\&Itemid=66). Rabatel, de l'équipe ICAR (Université de Lyon 2), adopte une approche énonciative. Les membres du Diltec (Université Paris 3) travaillent sur l'agir professionnel et ils ont recours à l'anthropologie pour étudier la didactique des langues (http.... (http://www.univ-paris3.fr/diltec-didactique-des-langues-des-textes-et-des- culturesea-2288-3451.kjsp). L'équipe Dylis de l'Université de Rouen noue étroitement recherches en sociolinguistique et recherches en didactique (http://dylis.univ-rouen.fr/content/présentationgénérale).

7. Comme contribuent à le faire Bautier (2001), Boutet (2017), Maingueneau (2003), von Münchow (2015), Hambye (2017).

8. Pour un bilan des travaux de Peytard, voir Madini (2010) et Moirand (2012). Cette dernière écrit p. 207 : «Jean Peytard est en ce sens um precursor no campo da linguistica discursiva, comme le dit le titre de ce colloque, une linguistique discursive fortement marquée par l'histoire de l'analyse sémiotique des textes littéraires (de Barthes à Kristeva), moins fortement, il me semble, par l'analyse du discours française des années 1970 (celle de Pêcheux, par exemple, mais aussi celle de Dubois, ou de Marcellesi, de Gardin), qu'il retrouvera dans les années 1980-1990. Cette linguistique du discours sera également marquée par l'importance de concepts venus d'ailleurs, de Bakhtine, de Vygotski, de Labov, de Jean-Blaise Grize ». Il s'agissait d'un colloque international organisé par l'Université Fédérale d'Ouro Preto et l'Université de Franche-Comté, Besançon à Ouro Preto: «Jean Peytard: syntagmes et entailles» dont «la thématique dominante fut l'articulation didactique-analyse de discours et l'articulation pratique professionnelle-pratique de recherche en relation avec la diffusion du français «Analyse du discours, Didactique, Enseignement du français ».

9. Centre de recherche sur les discours ordinaires et spécialisés.

10. Cf. présentation du numéro en ligne : https://cediscor.revues.org/345

11. Cf. http://eda.recherche.parisdescartes.fr/presentation/le-laboratoire-eda/: Les travaux «portent sur l'enseignement et l'apprentissage de la langue et plus largement du discours, en français (langue maternelle ou langue étrangère) et dans d'autres langues, et, d'autre part, les recherches en analyse de discours ou qui convoquent de l'analyse de discours portant 
directement sur les savoirs et sur leur transmission, notamment tels qu'ils apparaissent dans les programmes ou dans les manuels scolaires. Des recherches sur le lexique sont menées aussi bien dans la perspective de la didactique des langues que de l'analyse de discours. »

12. Cf. https://lab-e3d.u-bordeaux.fr/Axes-de-recherche/Langage-et-apprentissagesdisciplinaires: "Les chercheurs de l'équipe E3D, didacticiens de différentes disciplines, s'inscrivent dans le cadre d'une théorie qui attribue au langage une fonction essentielle dans la construction et la transformation de l'activité, des contextes de travail et des connaissances. [...] Les travaux conduits par les chercheurs membres de Lab-E3D, reposent ainsi sur l'idée d'une coconstruction des savoirs et des langages. Les principales notions développées actuellement par l'équipe et mises au travail dans ces recherches portent sur les contextes, la "secondarisation" des discours, le positionnement énonciatif et les communautés discursives disciplinaires scolaires. »

13. Et aussi : Le manuel est « un vecteur important de la scolarisation, conçue ici comme l'intégration de l'enfant dans le monde scolaire, espace-temps régi par des règles particulières. Il transmet des informations sur les connaissances jugées nécessaires par l'institution, et surtout, dans la manière de les présenter et de les organiser, il forme l'enfant aux méthodes et au langage scolaires. »

14. L'école est «cet espace qui leur dispense un enseignement. Or cet enseignement, tel qu'on peut l'appréhender dans les manuels, repose sur quelques lignes de sens qui, continuellement, produisent sous des formes multiples la figure de l'école comme le couronnement de toute chose. » (Maingueneau 1983 : 100).

15. Ainsi, par exemple, on s'est limité à la présentation de recherches qualitatives.

\section{AUTEUR \\ PASCALE DELORMAS}

Université Paris-Est, Céditec (EA 3119) 